\title{
Generating random networks that consist of a single connected component with a given degree distribution
}

\author{
Ido Tishby, Ofer Biham, and Eytan Katzav \\ Racah Institute of Physics, The Hebrew University, Jerusalem, 91904, Israel \\ Reimer Kühn \\ Mathematics Department, King's College London, \\ Strand, London WC2R 2LS, United Kingdom
}

\begin{abstract}
We present a method for the construction of ensembles of random networks that consist of a single connected component with a given degree distribution. This approach extends the construction toolbox of random networks beyond the configuration model framework, in which one controls the degree distribution but not the number of components and their sizes. Unlike configuration model networks, which are completely uncorrelated, the resulting single-component networks exhibit degree-degree correlations. Moreover, they are found to be disassortative, namely high-degree nodes tend to connect to low-degree nodes and vice versa. We demonstrate the method for singlecomponent networks with ternary, exponential and power-law degree distributions.
\end{abstract}

PACS numbers: $64.60 . \mathrm{aq}, 89.75 . \mathrm{Da}$ 


\section{INTRODUCTION}

Network models provide a useful description of a broad range of phenomena in the natural sciences and engineering as well as in the economic and social sciences. This realization has stimulated increasing interest in the structure of complex networks, and in the dynamical processes that take place on them [1 9]. One of the central lines of inquiry has been concerned with the existence of a giant connected component that is extensive in the network size. In the case of Erdős-Rényi (ER) networks, the critical parameters for the emergence of a giant component in the thermodynamic limit were identified and the fraction of nodes that reside in the giant component was determined [10 13]. These studies were later extended to the broader class of configuration model networks [14, 15]. The configuration model framework enables one to construct an ensemble of random networks whose degree sequences are drawn from a desired degree distribution, with no degree-degree correlations. The resulting network ensemble is a maximum entropy ensemble under the condition of the given degree distribution. A simple example of a configuration model network is the random regular graph, in which all the nodes are of the same degree, $k=c$. For random regular graphs with $c \geq 3$ the giant component encompasses the whole network [16]. However, in general, configuration model networks often exhibit a coexistence between a giant component, which is extensive in the network size, and many finite components, which are non-extensive trees. This can be exemplified by the case of ER networks, which exhibit a Poisson degree distribution of the form

$$
P(k)=\frac{e^{-c} c^{k}}{k !}
$$

where $c=\langle K\rangle$ is the mean degree. ER networks with $0<c<1$ consist of finite tree components. At $c=1$ there is a percolation transition, above which the network exhibits a coexistence between the giant component and the finite components. In the asymptotic limit, the size of the giant component is $N_{1}=g N$, where $N$ is the size of the whole network and the parameter $g=g(c)$, which vanishes for $c \leq 1$, increases monotonically for $c>1$. At $c=$ $\ln N$ there is a second transition, above which the giant component encompasses the entire

network [16]. In the range of $1<c<\ln N$, where the giant and finite components coexist, the structure and statistical properties of the giant component differ significantly from those of the whole network. In particular, the degree distribution of the giant component differs 
from $P(k)$ and it exhibits degree-degree correlations.

Recently, we developed a theoretical framework for the analytical calculation of the degree distribution and the degree-degree correlations in the giant component of configuration model networks [17]. In particular, this framework provides an analytical expression for the degree distribution of the giant component, denoted by $P(k \mid 1)$, in terms of the degree distribution $P(k)$ of the whole network. We applied this approach to the most commonly studied configuration model networks, namely with Poisson, exponential and power-law degree distributions. We have shown that the degree distribution of the giant component enhances the weight of the high-degree nodes and depletes the low-degree nodes, with respect to the whole network. Moreover, we found that the giant component is disassortative, namely high-degree nodes preferentially connect to low-degree nodes and vice versa. This appears to be a crucial feature that helps to maintain the integrity of the giant component.

In this paper we introduce a method for the construction of ensembles of random networks that consist of a single connected component with a given degree distribution, $P(k \mid 1)$. This is done by inverting the equations that express the degree distribution of the giant component $P(k \mid 1)$ in terms of the degree distribution $P(k)$ of the whole network. Constructing a configuration model network with the degree distribution $P(k)$ obtained from the inversion process, its giant component is found to exhibit the desired degree distribution $P(k \mid 1)$. We apply this approach to the construction of ensembles of random networks that consist of a single connected component with ternary, exponential and a power-law degree distributions.

The paper is organized as follows. In Sec. II we present the configuration model network ensemble. In Sec. III we present a method for the construction of a single-component network with a given degree distribution. In Sec. IV we analyze the properties of the resulting single-component networks. In particular, we present analytical expressions for the degree-degree correlations and the assortativity coefficient. In Sec. V we apply this methodology for the construction of networks that consist of a single connected component and exhibit ternary, exponential and power-law distributions. The results are discussed in Sec. VI and summarized in Sec. VII. 


\section{THE CONFIGURATION MODEL}

The configuration model network ensemble is an ensemble of uncorrelated random networks whose degree sequences are drawn from a given degree distribution, $P(k)$. In theoretical studies one often considers the asymptotic case in which the network size is infinite. In computer simulations, the network size $N$ is finite and the degree distribution is bounded from above and below such that $k_{\min } \leq k \leq k_{\max }$. For example, the commonly used choice of $k_{\text {min }}=1$ eliminates the possibility of isolated nodes in the network. Choosing $k_{\min }=2$ also eliminates the leaf nodes. Controlling the upper bound is important in the case of finite networks with degree distributions that exhibit fat tails, such as power-law degree distributions.

The configuration model ensemble is a maximum entropy ensemble under the condition that the degree distribution $P(k)$ is imposed [5, 18]. In this paper we focus on the case of undirected networks. To generate a network instance drawn from an ensemble of configuration model networks of $N$ nodes, with a given degree distribution $P(k)$, one draws the degrees of the $N$ nodes independently from $P(k)$. This gives rise to a degree sequence of the form $k_{1}, k_{2}, \ldots, k_{N}$ (where $\sum k_{i}$ must be even). Configuration model networks do not exhibit degree-degree correlations, which means that the conditional degree distribution of random neighbors of a random node of degree $k$ satisfies $P\left(k^{\prime} \mid k\right)=k^{\prime} P\left(k^{\prime}\right) /\langle K\rangle$ and does not depend on $k$. Also, the local structure of the network around a random node is typi-

cally a tree structure. A central feature of configuration model networks and other random networks above the percolation transition is the small-world property, namely the fact that the mean distance scales like $\langle L\rangle \sim \ln N$. Moreover, it was shown that scale-free networks for which $P(k) \propto k^{-\gamma}$ may be ultrasmall, depending on the exponent $\gamma$. In particular, for $2<\gamma<3$ their mean distance scales like $\langle L\rangle \sim \ln \ln N[19]$.

Configuration model networks in which $k_{\min }=1$ exhibit three different phases. In the sparse network limit, below the percolation transition, they consist of many finite tree components. Above the percolation transition there is a coexistence of a giant component and finite tree components. In the dense network limit there is a second transition, above which the giant component encompasses the whole network. In this paper we focus on the intermediate domain in which the giant and finite components coexist. The size of the giant component is determined by the degree distribution $P(k)$. 


\section{A. The construction of configuration model networks}

For the computer simulations presented below, we draw random network instances from an ensemble of configuration model networks of $N$ nodes, which follow a given degree distribution, $P(k)$. For each network instance we generate a degree sequence of the form $k_{1}, k_{2}, \ldots, k_{N}$, as described above. For the discussion below it is convenient to list the degree sequence in a decreasing order of the form $k_{1} \geq k_{2} \geq \cdots \geq k_{N}$.

It turns out that not every possible degree sequence is graphic, namely admissible as a degree sequence of a network. Therefore, before trying to construct a network with a given degree sequence, one should first confirm the graphicality of the degree sequence. To be graphic, a degree sequence must satisfy two conditions. The first condition is that the sum

of the degrees is an even number, namely $\sum_{i=1}^{N} k_{i}=2 L$, where $L$ is an integer that represents the number of edges in the network. The second condition is expressed by the Erdös-Gallai theorem, which states that an ordered sequence of the form $k_{1} \geq k_{2} \geq \cdots \geq k_{N}$ is graphic if and only if the condition

$$
\sum_{i=1}^{n} k_{i} \leq n(n-1)+\sum_{i=n+1}^{N} \min \left(k_{i}, n\right)
$$

holds for all values of $n$ in the range $1 \leq n \leq N-1$ [20, 21].

A convenient way to construct a configuration model network is to prepare the $N$ nodes such that each node $i$ is connected to $k_{i}$ half edges or stubs [5]. At each step of the construction, one connects a random pair of stubs that belong to two different nodes $i$ and $j$ that are not already connected, forming an edge between them. This procedure is repeated until all the stubs are exhausted. The process may get stuck before completion in a case in which all the remaining stubs belong to the same node or to pairs of nodes that are already connected. In such case one needs to perform some random reconnections in order to complete the construction.

\section{B. The degree distribution of the giant component}

Consider a configuration model network of $N$ nodes with a degree distribution, $P(k)$. To obtain the probability $g$ that a random node in the network belongs to the giant component, one needs to first calculate the probability $\tilde{g}$, that a random neighbor of a random node, $i$, 
belongs to the giant component of the reduced network that does not include the node $i$. The probability $\tilde{g}$ is determined by [6]

$$
1-\tilde{g}=G_{1}(1-\tilde{g})
$$

where

$$
G_{1}(x)=\sum_{k=1}^{\infty} x^{k-1} \widetilde{P}(k)
$$

is the generating function of $\widetilde{P}(k)$, and

$$
\widetilde{P}(k)=\frac{k}{\langle K\rangle} P(k)
$$

is the degree distribution of nodes that are sampled as random neighbors of random nodes. Using $\tilde{g}$, one can then obtain the probability $g$ from the equation

$$
g=1-G_{0}(1-\tilde{g})
$$

where

$$
G_{0}(x)=\sum_{k=0}^{\infty} x^{k} P(k)
$$

is the generating function of $P(k)$. Given that $G_{0}(x)$ and $G_{1}(x)$, defined by Eqs. (7) and (41), respectively, are probability generating functions, they satisfy $G_{0}(1)=G_{1}(1)=1$. This property entails that $\tilde{g}=0$ is always a solution of $\mathrm{Eq}(\underline{3})$. This (trivial) solution implies $g=0$ and describes a subcritical network, in which case the key question is, whether other solutions with $\tilde{g}>0$, hence $g>0$, exist as well.

In configuration model networks that do not include any isolated nodes (of degree $k=$ 0 ) and leaf nodes (of degree $k=1$ ), namely $k_{\text {min }} \geq 2$, the generating functions satisfy $G_{0}(0)=0$ and $G_{1}(0)=0$. This solution corresponds to the case where the giant component encompasses the whole network and $g=\tilde{g}=1$. This implies that in such networks both $x=0$ and $x=1$ are fixed points of both $G_{0}(x)$ and $G_{1}(x)$. Furthermore, it can be shown that in networks whose degree distributions satisfy the condition that $k_{\min } \geq 2$ and $k_{\max } \geq 3$ there are no other (nontrivial) fixed points for $G_{0}(x)$ and $G_{1}(x)$ with $0<x<1$ [22]. This means that in such networks the giant component encompasses the whole network. Here we 
are interested in configuration model networks that exhibit a coexistence between the giant and the finite components. Such coexistence appears for degree distributions that support a non-trivial solution of Eq. (3) , in which $0<\tilde{g}<1$. A necessary condition for such solution is the existence of leaf nodes of degree $k=1$, namely, $P(1)>0$. Therefore, we focus here on degree distributions in which $k_{\min }=1$.

For the analysis presented below we introduce an indicator variable $\Lambda \in\{0,1\}$, where $\Lambda=1$ indicates that an event takes place on the giant component and $\Lambda=0$ indicates that it happens on one of the finite components. In this notation, the probability that a random node resides on the giant component is $P(\Lambda=1)=g$, and the probability that it resides on one of the finite components is $P(\Lambda=0)=1-g$. Similarly, the probabilities that a random neighbor of a random node resides on the giant component is $\widetilde{P}(\Lambda=1)=\tilde{g}$ and the probability that it resides on one of the finite components is $\widetilde{P}(\Lambda=0)=1-\tilde{g}$.

A node, $i$, of degree $k$ resides on the giant component if at least one of its $k$ neighbors resides on the giant component of the reduced network from which $i$ is removed. Therefore, the probability $g_{k}$ that a random node of degree $k$ resides on the giant component is given by

$$
g_{k}=P(\Lambda=1 \mid k)=1-(1-\tilde{g})^{k},
$$

while the probability that such node resides on one of the finite components is

$$
P(\Lambda=0 \mid k)=1-g_{k}=(1-\tilde{g})^{k}
$$

Using Bayes' theorem, one can show that the degree distribution, conditioned on the giant component, is given by [17]

$$
P(k \mid \Lambda=1)=\frac{1-(1-\tilde{g})^{k}}{g} P(k),
$$

while the degree distribution, conditioned on the finite components, is given by

$$
P(k \mid \Lambda=0)=\frac{(1-\tilde{g})^{k}}{1-g} P(k) .
$$

The mean degree of the giant component is

$$
\mathbb{E}[K \mid \Lambda=1]=\frac{1-(1-\tilde{g})^{2}}{g}\langle K\rangle,
$$


while the mean degree on the finite components is

$$
\mathbb{E}[K \mid \Lambda=0]=\frac{(1-\tilde{g})^{2}}{1-g}\langle K\rangle,
$$

where

$$
\langle K\rangle=\sum_{k=0}^{\infty} k P(k)
$$

is the mean degree of the whole network. In the rest of the paper, for the sake of brevity, we will drop the indicator $\Lambda$ and use $P(k \mid 0)$ and $P(k \mid 1)$ to denote the degree distribution on the finite components and on the giant component, respectively. Similarly, we will use $\mathbb{E}[K \mid 0]$ $(\mathbb{E}[K \mid 1])$ to denote the expected degree on the finite (giant) component. It is interesting to mention that just above the percolation transition, when the giant component just emerges, $\mathbb{E}[K \mid 1] \rightarrow 2[17,23]$. This will be important in the rest of the paper, because it means that if one wants to generate a network that forms a single component with a given degree distribution $P(k \mid 1)$, the mean of this distribution must satisfy $\mathbb{E}[K \mid 1] \geq 2$. From a different angle, a single tree component of $N$ nodes satisfies $\mathbb{E}[K \mid 1]=2-2 / N[24]$, thus $\mathbb{E}[K \mid 1] \rightarrow 2$ in the asymptotic limit. Above the percolation transition cycles start to emerge in the giant component, and $\mathbb{E}[K \mid 1]$ gradually increases. As the network becomes more dense, the fraction of nodes, $g$, that reside on the giant component increases. When $g \rightarrow 1$ the giant component encompasses the whole network. The value of $\mathbb{E}[K \mid 1]$ at which $g \rightarrow 1$ depends on the degree distribution.

\section{The size of the giant component}

The expectation value of the size of the giant component of a configuration model of $N$ nodes with a degree distribution $P(k)$ is given by

$$
\left\langle N_{1}\right\rangle=N g
$$

where $g$ is given by Eq. (6). However, in any single network instance the size $N_{1}$ of the giant component may deviate from $\left\langle N_{1}\right\rangle$. Below we consider the distribution $P\left(N_{1}\right)$ of the

sizes of the giant components obtained in an ensemble of configuration model networks of $N$ nodes with degree distribution $P(k)$. To get a rough idea about the form of $P\left(N_{1}\right)$, one 
may assume, for simplicity, that each node independently resides on the giant component with probability $g$, with no correlations between different nodes. In such case, $P\left(N_{1}\right)$ would follow a binomial distribution that converges to a Gaussian distribution whose mean is given by Eq. (15). The variance of such a distribution is given by

$$
\operatorname{Var}\left(N_{1}\right)=N \sum_{k=1}^{N-1} g_{k}\left(1-g_{k}\right) P(k),
$$

where $g_{k}$ is given by Eq. (8). In dense networks that exhibit a narrow degree distribution, such that $g_{k}$ is only weakly dependent on $k$, Eq. (16) can be approximated by

$$
\operatorname{Var}\left(N_{1}\right)=N g(1-g) .
$$

In the case of ER networks [25, 26], in which $P(k)$ is a Poisson distribution, as in Eq. (11), it was shown that $P\left(N_{1}\right)$ is a Gaussian distribution whose mean is given by Eq. (15) and its variance is given by

$$
\operatorname{Var}\left(N_{1}\right)=\frac{N g(1-g)}{1-\langle K\rangle(1-g)} .
$$

For configuration model networks with other degree distributions there are rigorous results for the size distribution of the giant component only in the weakly supercritical range [26, 27], which is just above the percolation phase transition. More precisely, in configuration model networks the percolation transition follows the Molloy-Reed criterion [14, 15], namely, it takes place at $\langle K(K-1)\rangle /\langle K\rangle=1$. Just above the transition, in the limit $\epsilon=\langle K(K-$ $1)\rangle /\langle K\rangle-1 \rightarrow 0^{+}$, the distribution $P\left(N_{1}\right)$ is a Gaussian distribution whose mean is

$$
\left\langle N_{1}\right\rangle=\frac{2\langle K\rangle^{2}}{\langle K(K-1)(K-2)\rangle} \epsilon N
$$

and its variance is given by

$$
\operatorname{Var}\left(N_{1}\right)=\frac{2\langle K\rangle}{\epsilon} N .
$$

This means that at the percolation transition the variance of $N_{1}$ diverges, and starts decreasing above the transition. There are no rigorous results in the full supercritical range, but following the ER case, it is plausible that the normality of $P\left(N_{1}\right)$ still holds, at least for a degree distribution $P(k)$ with a finite variance, while the variance $\operatorname{Var}\left(N_{1}\right)$ decreases. The 
main conclusion of this discussion is that sufficiently far above the percolation transition, where the giant component is not too small, the size fluctuations of the giant component become negligible as $N$ is increased.

\section{THE CONSTRUCTION OF A SINGLE-COMPONENT NETWORK WITH A GIVEN DEGREE DISTRIBUTION}

Here we present a method for the construction of a network that consists of a single component whose degree sequence is effectively drawn from a given degree distribution, denoted by $P(k \mid 1)$. The approach is based on the construction of a configuration model network whose degree sequence is drawn from a suitable degree distribution $P(k)$, such that its giant component exhibits the desired degree distribution, $P(k \mid 1)$.

Inverting Eq. (10) we find that in order to obtain a giant component whose degree distribution is $P(k \mid 1)$, the degree distribution of the whole network should be

$$
P(k)=\frac{g}{1-(1-\tilde{g})^{k}} P(k \mid 1),
$$

where $\tilde{g}$ is given by Eq. (3) and $g$ is given by Eq. (66). The mean degree of the whole network will thus be

$$
\langle K\rangle=\sum_{k=1}^{\infty} \frac{g k}{1-(1-\tilde{g})^{k}} P(k \mid 1) .
$$

In order to obtain an ensemble of single-component networks whose mean size is $\left\langle N_{1}\right\rangle$, the size of the configuration model networks from which these giant components are obtained should be

$$
N=\frac{\left\langle N_{1}\right\rangle}{g}
$$

For the analysis below it is useful to introduce the generating functions for the degree distribution conditioned on the giant component, namely

$$
G_{0}^{1}(x)=\sum_{k=1}^{\infty} x^{k} P(k \mid 1)
$$

and 


$$
G_{1}^{1}(x)=\sum_{k=1}^{\infty} \frac{k x^{k-1}}{\mathbb{E}[K \mid 1]} P(k \mid 1) .
$$

These generating functions are related to each other by the equation

$$
G_{1}^{1}(x)=\frac{\frac{d}{d x} G_{0}^{1}(x)}{\left.\frac{d}{d x} G_{0}^{1}(x)\right|_{x=1}} .
$$

In order to calculate the probability $\tilde{g}$, we utilize Eq. (3), where we express $P(k)$ and $\langle K\rangle$ in terms of $P(k \mid 1)$, and obtain

$$
1-\tilde{g}=\frac{\sum_{k=1}^{\infty} \frac{k(1-\tilde{g})^{k-1}}{1-(1-\tilde{g})^{k}} P(k \mid 1)}{\sum_{k=1}^{\infty} \frac{k}{1-(1-\tilde{g})^{k}} P(k \mid 1)} .
$$

Using the Taylor expansion of $(1-x)^{-1}$, which takes the form

$$
\frac{1}{1-x}=\sum_{n=0}^{\infty} x^{n}
$$

where $0<x<1$, to express the term $1 /\left[1-(1-\tilde{g})^{k}\right]$ as a power series in $(1-\tilde{g})^{k}$, we obtain

$$
1-\tilde{g}=\frac{\sum_{k=1}^{\infty} k(1-\tilde{g})^{k-1} \sum_{n=0}^{\infty}(1-\tilde{g})^{k n} P(k \mid 1)}{\sum_{k=1}^{\infty} k \sum_{n=0}^{\infty}(1-\tilde{g})^{k n} P(k \mid 1)} .
$$

Multiplying both sides by $1-\tilde{g}$ and exchanging the order of summations in the numerator and denominator, we obtain

$$
(1-\tilde{g})^{2}=\frac{\sum_{n=1}^{\infty}(1-\tilde{g})^{n} \sum_{k=1}^{\infty} k(1-\tilde{g})^{n(k-1)} P(k \mid 1)}{\sum_{n=0}^{\infty}(1-\tilde{g})^{n} \sum_{k=1}^{\infty} k(1-\tilde{g})^{n(k-1)} P(k \mid 1)} .
$$

Adding and subtracting the $n=0$ term in the numerator, this equation can be expressed in the form

$$
(1-\tilde{g})^{2}=1-\frac{\mathbb{E}[K \mid 1]}{\sum_{n=0}^{\infty}(1-\tilde{g})^{n} \sum_{k=1}^{\infty} k(1-\tilde{g})^{n(k-1)} P(k \mid 1)} .
$$

Using the generating function $G_{1}^{1}(x)$, Eq. (31) can be written in the form 


$$
(1-\tilde{g})^{2}=1-\frac{1}{\sum_{n=0}^{\infty}(1-\tilde{g})^{n} G_{1}^{1}\left[(1-\tilde{g})^{n}\right]}
$$

or in the form

$$
\tilde{g}(2-\tilde{g}) \sum_{n=0}^{\infty}(1-\tilde{g})^{n} G_{1}^{1}\left[(1-\tilde{g})^{n}\right]=1 .
$$

This is an implicit equation that should be solved in order to obtain the parameter $\tilde{g}$. For some degree distributions one can obtain a closed form analytical expression for $\tilde{g}$, while for other distributions it should be calculated numerically. A useful approximation scheme would be to replace the sum in Eq. (33) by an integral. To improve the accuracy of this approximation, it is useful to first separate the $n=0$ and the $n=1$ terms from the rest of the sum and obtain

$$
\tilde{g}(2-\tilde{g})\left[1+(1-\tilde{g}) G_{1}^{1}(1-\tilde{g})+\sum_{n=2}^{\infty}(1-\tilde{g})^{n} G_{1}^{1}\left[(1-\tilde{g})^{n}\right]\right]=1 .
$$

Using Eq. (26) we find that

$$
x^{n} G_{1}^{1}\left(x^{n}\right)=\frac{\frac{\partial}{\partial n}\left[G_{0}^{1}\left(x^{n}\right)\right]}{\mathbb{E}[K \mid 1] \ln x} .
$$

Replacing the sum $\sum_{n=2}^{\infty}$ in Eq. (34) by an integral of the form $\int_{3 / 2}^{\infty} d n$ and carrying out the integration using Eq. (35), we obtain

$$
\tilde{g}(2-\tilde{g})\left[1+(1-\tilde{g}) G_{1}^{1}(1-\tilde{g})-\frac{G_{0}^{1}\left[(1-\tilde{g})^{3 / 2}\right]}{\mathbb{E}[K \mid 1] \ln (1-\tilde{g})}\right]=1 .
$$

This equation is easier to handle than Eq. (33), although usually it can be solved only numerically. Other, more precise schemes, could be devised by treating more individual terms of the sum in Eq. (33) separately, say up to $n=2$ or $n=3$, and approximating the tail of the sum by an integral. Our experience tells us that for the cases considered in this paper using the $n=1$ scheme provides values of $\tilde{g}$ that differ by at most a few percents from the exact value.

Once the parameter $\tilde{g}$ is known, the parameter $g$ can be obtained from

$$
1-g=\sum_{k=0}^{\infty} \frac{g(1-\tilde{g})^{k}}{1-(1-\tilde{g})^{k}} P(k \mid 1)
$$


Extracting $g$ we obtain

$$
g=\frac{1}{1+\sum_{k=0}^{\infty} \frac{(1-\tilde{g})^{k}}{1-(1-\tilde{g})^{k}} P(k \mid 1)}
$$

Expanding the denominator according to Eq. (28) and exchanging the order of the summations, we obtain

$$
g=\frac{1}{1+\sum_{n=1}^{\infty} G_{0}^{1}\left[(1-\tilde{g})^{n}\right]} .
$$

To conclude, in order to obtain an ensemble of single-component networks whose mean size is $\left\langle N_{1}\right\rangle$, with degree sequences that are effectively drawn from $P(k \mid 1)$, one constructs an ensemble of configuration model networks whose size $N$ is given by Eq. (23) and its degree distribution $P(k)$ is given by Eq. (21). The giant components of these networks are the desired single component networks. The mean degree $\langle K\rangle$ of the configuration model networks is

$$
\langle K\rangle=\frac{g}{1-(1-\tilde{g})^{2}} \mathbb{E}[K \mid 1] .
$$

Note that it is also possible to control the exact size of the single-component network. Consider the case in which the desired size of a given instance of the single-component network is $\left\lfloor\left\langle N_{1}\right\rangle\right\rfloor$, namely the integer part of $\left\langle N_{1}\right\rangle$. In a case in which the size of the giant component $n_{1}$ came out smaller than $\left\lfloor\left\langle N_{1}\right\rangle\right\rfloor$, one should add nodes to the configuration model network until the giant component will reach the desired size. The degrees of the added nodes are drawn from $P(k)$. To add a node of an even degree $k$ to the network one picks randomly $k / 2$ edges that connect $k$ distinct nodes. One then cuts each edge in the middle to generate $k$ stubs. The $k$ stubs of the new node are then connected to these $k$ stubs. In the case of nodes of odd degrees, $k$ and $k^{\prime}$, one picks randomly $\left(k+k^{\prime}\right) / 2$ edges and cuts them in the middle to generate $k+k^{\prime}$ stubs. The stubs of the two new nodes are then connected randomly to these $k+k^{\prime}$ stubs. In a case in which $n_{1}$ came out larger than $\left\lfloor\left\langle N_{1}\right\rangle\right\rfloor$ one should delete random nodes (one at a time for even-degree nodes and in pairs for odd-degree nodes), until the giant component is reduced to the desired size. The open stubs that remain from the edges of each deleted node are then randomly connected to each other in pairs. 


\section{PROPERTIES OF SINGLE COMPONENT RANDOM NETWORKS}

Unlike configuration model networks that are completely uncorrelated, their giant components exhibit degree-degree correlations. In particular, following the observation made in Ref. [17] that the giant components are disassortative, below we prove this property. Interestingly, this observation has been recently demonstrated in percolating clusters [28].

The joint degree distribution of a pair of adjacent nodes in a configuration model network with degree distribution $P(k)$ is given by [17]

$$
\widehat{P}\left(k, k^{\prime} \mid 1\right)=\frac{1-(1-\tilde{g})^{k+k^{\prime}-2}}{1-(1-\tilde{g})^{2}} \frac{k}{\langle K\rangle} P(k) \frac{k^{\prime}}{\langle K\rangle} P\left(k^{\prime}\right) .
$$

Expressing $P(k)$ and $P\left(k^{\prime}\right)$ in terms of $P(k \mid 1)$ and $P\left(k^{\prime} \mid 1\right)$, respectively, using Eq. (21), we obtain

$$
\widehat{P}\left(k, k^{\prime} \mid 1\right)=W\left(k, k^{\prime}\right) \frac{k}{\mathbb{E}[k \mid 1]} P(k \mid 1) \frac{k^{\prime}}{\mathbb{E}[k \mid 1]} P\left(k^{\prime} \mid 1\right),
$$

where

$$
W\left(k, k^{\prime}\right)=\tilde{g}(2-\tilde{g}) \frac{1-(1-\tilde{g})^{k+k^{\prime}-2}}{\left[1-(1-\tilde{g})^{k}\right]\left[1-(1-\tilde{g})^{k^{\prime}}\right]}
$$

accounts for the degree-degree correlations between adjacent nodes. For example, $W(1,1)=$ 0 , reflecting the fact that pairs of nodes of degree $k=1$ on the giant component cannot share an edge, because in such case they will form an isolated dimer. Also, one can verify that $W(k, 2)=1$ for all values of $k \geq 1$. This means that nodes of degree $k=2$ are distributed randomly in the giant component and are not correlated to the degrees of their neighboring nodes. The degree-degree correlations between nodes of degree $k \geq 3$ and leaf nodes of degree $k^{\prime}=1$ is given by

$$
W(k, 1)=1+\frac{1-\tilde{g}-(1-\tilde{g})^{k-1}}{1-(1-\tilde{g})^{k}}>1 .
$$

Thus, there is a positive correlation between leaf nodes and nodes of degree $k \geq 3$. Moreover, the correlation becomes stronger as $k$ increases.

Below we show that $W\left(k, k^{\prime}\right) \leq 1$ for for $k, k^{\prime} \geq 3$, hence the degree-degree correlations

between pairs of nodes of degrees $k, k^{\prime} \geq 3$ are negative. To this end we denote $\tilde{h}=1-\tilde{g}$, which satisfies $0<\tilde{h}<1$. Expressing $W\left(k, k^{\prime}\right)$ in terms of $\tilde{h}$, we obtain 


$$
W\left(k, k^{\prime} ; \tilde{h}\right)=\left(1-\tilde{h}^{2}\right) \frac{1-\tilde{h}^{k+k^{\prime}-2}}{\left(1-\tilde{h}^{k}\right)\left(1-\tilde{h}^{k^{\prime}}\right)} .
$$

The diagonal terms, obtained for $k=k^{\prime}$, are given by

$$
f(k ; \tilde{h})=W(k, k ; \tilde{h})=\left(1-\tilde{h}^{2}\right) \frac{1-\tilde{h}^{2 k-2}}{\left(1-\tilde{h}^{k}\right)^{2}} .
$$

For $k=3$ we obtain

$$
f(k=3 ; \tilde{h})=\frac{(1+\tilde{h})^{2}\left(1+\tilde{h}^{2}\right)}{\left(1+\tilde{h}+\tilde{h}^{2}\right)^{2}} .
$$

Differentiating $f(k=3 ; \tilde{h})$ with respect to $\tilde{h}$, we obtain

$$
\frac{\partial}{\partial \tilde{h}} f(k=3 ; \tilde{h})=-\frac{2 \tilde{h}\left(1-\tilde{h}^{2}\right)}{\left(1+\tilde{h}+\tilde{h}^{2}\right)^{3}}<0,
$$

for $0<\tilde{h}<1$. Therefore, the function $f(k=3 ; \tilde{h})$ is a monotonically decreasing function of $\tilde{h}$. This implies that

$$
f(k=3 ; \tilde{h}) \leq f(k=3 ; \tilde{h}=0)=1,
$$

with equality taking place only at $\tilde{h}=0$. Considering the degree, $k$, as a continuous variable and taking the derivative of $f(k ; \tilde{h})$ with respect to $k$, we obtain

$$
\frac{\partial}{\partial k} f(k ; \tilde{h})=-\frac{2 \tilde{h}^{k}\left(1-\tilde{h}^{2}\right)\left(1-\tilde{h}^{k-2}\right) \ln \left(\frac{1}{\tilde{h}}\right)}{\left(1-\tilde{h}^{k}\right)^{3}}<0
$$

for $k>2$ and $0<\tilde{h}<1$. This means that $f(k ; \tilde{h})$ is a monotonically decreasing function in both $k$ and $\tilde{h}$. We thus conclude that $W(k, k)<1$ for all values of $k \geq 3$ and $0<\tilde{h}<1$. In order to show that $W\left(k, k^{\prime}\right)<1$ for all $k, k^{\prime} \geq 3$, it is sufficient to show that under these conditions $W\left(k, k^{\prime}\right)$ is a monotonically decreasing function of $k^{\prime}$ for all values of $0<\tilde{h}<1$. This is shown by differentiating $W\left(k, k^{\prime} ; \tilde{h}\right)$ with respect to $k^{\prime}$, which leads to

$$
\frac{\partial}{\partial k^{\prime}} W\left(k, k^{\prime} ; \tilde{h}\right)=-\frac{\tilde{h}^{k^{\prime}}\left(1-\tilde{h}^{2}\right)\left(1-\tilde{h}^{k-2}\right) \ln \left(\frac{1}{\tilde{h}}\right)}{\left(1-\tilde{h}^{k}\right)\left(1-\tilde{h}^{k^{\prime}}\right)^{2}}<0
$$

where $k>2$ and $0<\tilde{h}<1$. This means that for any combination of $k, k^{\prime} \geq 3$, where $k^{\prime}>k$, the correlation function $W\left(k, k^{\prime}\right)$ satisfies $W\left(k, k^{\prime}\right)<W(k, k)<1$. We thus conclude that pairs of adjacent nodes of degrees $k, k^{\prime} \geq 3$ exhibit negative degree-degree correlations. 
The probability that a node connected to a random edge in the giant component is of degree $k$ is given by [17]

$$
\widehat{P}(k \mid 1)=\frac{k}{\mathbb{E}[K \mid 1]} P(k \mid 1) .
$$

The assortativity coefficient [29] of the giant component is given by [17]

$$
r=\frac{\sum_{k, k^{\prime} \geq 2}(k-1)\left(k^{\prime}-1\right) \widehat{P}\left(k, k^{\prime} \mid 1\right)-\left[\sum_{k \geq 2}(k-1) \widehat{P}(k \mid 1)\right]^{2}}{\sum_{k \geq 2}(k-1)^{2} \widehat{P}(k \mid 1)-\left[\sum_{k \geq 2}(k-1) \widehat{P}(k \mid 1)\right]^{2}} .
$$

Since the degree-degree correlations between pairs of adjacent nodes of degrees $k, k^{\prime} \geq 3$ are negative, the assortativity coefficient of the giant component must satisfy $r<0$. This is an essential property of the giant components of configuration model networks, which is required in order to maintain the integrity of the giant component.

\section{APPLICATIONS TO SPECIFIC NETWORK MODELS}

In this section we apply the methodology developed above for the construction of networks that consist of a single connected component, with a prescribed degree distribution, $P(k \mid 1)$, for some popular ensembles of random networks.

\section{A. Construction of a single-component network with a ternary degree distribution}

The properties of the giant component of a random network are sensitive to the abundance of nodes of low degrees, particularly nodes of degree $k=1$ (leaf nodes) and $k=2$. Nodes of degree $k=0$ (isolated nodes) are excluded from the giant component and their weight in the degree distribution of the whole network has no effect on the properties of the giant component. Therefore, it is useful to consider a simple configuration model in which all nodes are restricted to a small number of low degrees. Here we consider a configuration model network with a ternary degree distribution of the form [5]

$$
P(k)=p_{1} \delta_{k, 1}+p_{2} \delta_{k, 2}+p_{3} \delta_{k, 3},
$$

where $\delta_{k, n}$ is the Kronecker delta, and $p_{1}+p_{2}+p_{3}=1$. The mean degree of such network is given by 


$$
\langle K\rangle=p_{1}+2 p_{2}+3 p_{3} .
$$

The generating functions of the degree distribution are

$$
G_{0}(x)=p_{1} x+p_{2} x^{2}+p_{3} x^{3},
$$

and

$$
G_{1}(x)=\frac{p_{1}+2 p_{2} x+3 p_{3} x^{2}}{p_{1}+2 p_{2}+3 p_{3}} .
$$

Solving Eq. (3) for $\tilde{g}$, with $G_{1}(x)$ given by Eq. (57), we find that

$$
\tilde{g}= \begin{cases}0 & p_{3} \leq \frac{p_{1}}{3} \\ 1-\frac{p_{1}}{3 p_{3}} & p_{3}>\frac{p_{1}}{3} .\end{cases}
$$

Using Eq. (66) to evaluate the parameter $g$, where $G_{0}(x)$ is given by Eq. (56), we find that

$$
g= \begin{cases}0 & p_{3} \leq \frac{p_{1}}{3} \\ 1-\left(\frac{p_{1}}{3 p_{3}}\right) p_{1}-\left(\frac{p_{1}}{3 p_{3}}\right)^{2} p_{2}-\left(\frac{p_{1}}{3 p_{3}}\right)^{3} p_{3} & p_{3}>\frac{p_{1}}{3} .\end{cases}
$$

Thus, the percolation threshold is located at $p_{3}=p_{1} / 3$. This can be understood intuitively by recalling that the finite components exhibit a tree structure. In a tree that includes a single node of degree $k=3$ there must be three leaf nodes of degree $k=1$. In the giant component, which includes cycles, there must be more than one node of degree 3 for every three nodes of degree 1 . This is not likely to occur in a case in which $p_{3}<p_{1} / 3$. Using the normalization condition, we find that for any given value of $p_{2}$, a giant component exists for $p_{3}>\left(1-p_{2}\right) / 4$.

Using Eq. (10), we obtain the degree distribution of the giant component, which is given by

$$
P(k \mid 1)=\left[\frac{1-\left(\frac{p_{1}}{3 p_{3}}\right)^{k}}{1-\left(\frac{p_{1}}{3 p_{3}}\right) p_{1}-\left(\frac{p_{1}}{3 p_{3}}\right)^{2} p_{2}-\left(\frac{p_{1}}{3 p_{3}}\right)^{3} p_{3}}\right] P(k),
$$

where $k=1,2,3$ and $P(k)$ is given by Eq. (54). 
These results enable us to construct a giant connected component with a desired ternary degree distribution, given by $P(k \mid 1), k=1,2,3$, where $\sum_{k=1}^{3} P(k \mid 1)=1$. To this aim, we need to express the degree distribution $P(k)$ of the whole network, given by Eq. (54), in terms of the given degree distribution $P(k \mid 1)$ of the giant component. We should first evaluate the parameter $\tilde{g}$, which is given by

$$
\tilde{g}=1-\frac{p_{1}}{3 p_{3}} .
$$

Using Eq. (60) to calculate the ratio $P(1 \mid 1) / P(3 \mid 1)$, we obtain

$$
\frac{P(1 \mid 1)}{3 P(3 \mid 1)}=\frac{1}{1+\left(\frac{p_{1}}{3 p_{3}}\right)+\left(\frac{p_{1}}{3 p_{3}}\right)^{2}} \frac{p_{1}}{3 p_{3}}
$$

Solving for $p_{1} /\left(3 p_{3}\right)$ we obtain

$$
\frac{p_{1}}{3 p_{3}}=\frac{1}{2}\left[\frac{3 P(3 \mid 1)}{P(1 \mid 1)}-1-\sqrt{\left(\frac{3 P(3 \mid 1)}{P(1 \mid 1)}+1\right)\left(\frac{3 P(3 \mid 1)}{P(1 \mid 1)}-3\right)}\right] .
$$

Therefore

$$
\tilde{g}=\frac{1}{2}\left[3-\frac{3 P(3 \mid 1)}{P(1 \mid 1)}+\sqrt{\left(\frac{3 P(3 \mid 1)}{P(1 \mid 1)}+1\right)\left(\frac{3 P(3 \mid 1)}{P(1 \mid 1)}-3\right)}\right] .
$$

The next step is to evaluate the parameter $g$, which is given by

$$
g=\frac{1}{1+\sum_{k=1}^{3} \frac{(1-\tilde{g})^{k}}{1-(1-\tilde{g})^{k}} P(k \mid 1)} .
$$

Simplifying the expression we obtain

$$
g=\frac{\tilde{g}}{P(1 \mid 1)+\frac{1}{2-\tilde{g}} P(2 \mid 1)+\frac{1}{3-3 \tilde{g}+\tilde{g}^{2}} P(3 \mid 1)} .
$$

Using the normalization condition of the probabilities $P(k \mid 1)$ to express $P(2 \mid 1)$ in terms of $P(1 \mid 1)$ and $P(3 \mid 1)$ we obtain

$$
g=\frac{\tilde{g}(2-\tilde{g})}{1+(1-\tilde{g}) P(1 \mid 1)-\frac{(1-\tilde{g})^{2}}{3-3 \tilde{g}+\tilde{g}^{2}} P(3 \mid 1)} .
$$

The degree distribution of the whole network is given by Eq. (54), where 


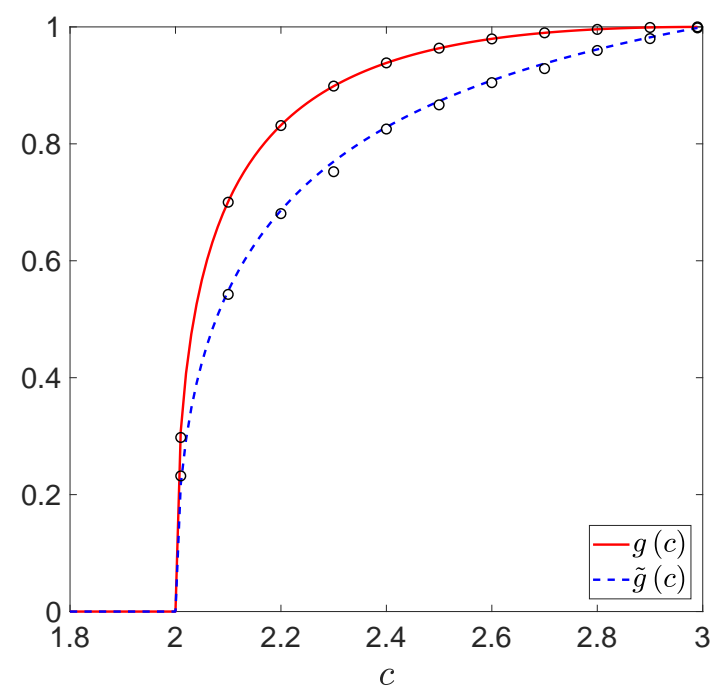

FIG. 1: (Color online) Analytical results for the fraction of nodes $g$ (solid line), and the fraction of random neighbors of random nodes, $\tilde{g}$ (dashed line), that reside on the giant component, in a configuration model network whose giant component exhibits a ternary degree distribution $P(k \mid 1)$, expressed by Eq. (60), with $P(K=2 \mid 1)=0$, as a function of the mean degree $c=\mathbb{E}[K \mid 1]$ of the giant component. The simulation results (circles), obtained for $N=10^{4}$, are in very good agreement with the analytical results.

$$
\begin{aligned}
& p_{1}=\frac{g}{\tilde{g}} P(1 \mid 1) \\
& p_{2}=\frac{g}{\tilde{g}(2-\tilde{g})} P(2 \mid 1) \\
& p_{3}=\frac{g}{\tilde{g}\left(3-3 \tilde{g}+\tilde{g}^{2}\right)} P(3 \mid 1) .
\end{aligned}
$$

Thus, in order to obtain an ensemble of single-component networks of mean size $\left\langle N_{1}\right\rangle$, whose degree sequences are drawn from a given ternary degree distribution $P(k \mid 1)$, one generates an ensemble of configuration model networks with a degree distribution $P(k)$, given by Eq. (54), where $p_{1}, p_{2}$ and $p_{3}$ are given by Eq. (68). The size of the configuration model networks should be $N=\left\langle N_{1}\right\rangle / g$, where $g$ is given by Eq. (66).

In Fig. 11we present analytical results for the probability $g$, obtained from Eq. (67), that a randomly selected node resides on the giant component (solid line), in a configuration model network whose giant component exhibits a ternary degree distribution with $P(K=2 \mid 1)=0$, as a function of the mean degree $c=\mathbb{E}[K \mid 1]$ of the giant component. We also show the 
probability $\tilde{g}$, obtained from Eq. (64), that a random neighbor of a random node resides on the giant component (dashed line). As discussed above, both $g$ and $\tilde{g}$ vanish for $c<2$, since there are no giant components with mean degrees smaller than 2 . For $c>2$ both $g$ and $\tilde{g}$ exhibit a steep rise as $c$ is increased, reaching $g=\tilde{g}=1$ at $c=3$, where the giant component encompasses the whole network. The results obtained from computer simulations (circles) with $N=10^{4}$ are found to be in very good agreement with the analytical results.

\section{B. Construction of a single component network with an exponential degree dis-} tribution

Consider a configuration model network whose giant component exhibits an exponential degree distribution of the form

$$
P(k \mid 1)=A e^{-\alpha k}
$$

where $k \geq k_{\min }$. Here we focus on the case of $k_{\min }=1$, for which the normalization factor is $A=e^{\alpha}-1$. The mean degree is given by

$$
c=\mathbb{E}[K \mid 1]=\frac{1}{1-e^{-\alpha}}
$$

For the analysis below, it is convenient to parametrize the degree distribution in terms of the mean degree $c$. Plugging in $\alpha=\ln c-\ln (c-1)$ we obtain

$$
P(k \mid 1)=\frac{1}{c}\left(\frac{c-1}{c}\right)^{k-1},
$$

where $k \geq 1$. The mean degree of nodes that reside on the giant component is $\mathbb{E}[K \mid 1]=c$. As noted above, a giant component exists only for $c \geq 2$. This implies that $\alpha$ must satisfy the condition $\alpha \leq \ln 2$. Inserting $P(k \mid 1)$ from Eq. (71) into Eqs. (24) and (25) and carrying out the summations, we find that the generating functions for a giant component with an exponential degree distribution take the form

$$
G_{0}^{1}(x)=\frac{x}{c-x(c-1)}
$$

and 


$$
G_{1}^{1}(x)=\frac{1}{[c+(1-c) x]^{2}} .
$$

Plugging in $x=(1-\tilde{g})^{n}$ in Eq. (73) and inserting the result into Eq. (33), we obtain that $\tilde{g}$ is given by

$$
\tilde{g}(2-\tilde{g}) \sum_{n=0}^{\infty} \frac{(1-\tilde{g})^{n}}{\left[c+(1-c)(1-\tilde{g})^{n}\right]^{2}}=1 .
$$

This is an implicit equation for $\tilde{g}$ in terms of the mean degree $c$, which is essentially equivalent to Eq. (33) for the case of the exponential distribution. It should be solved numerically in order to obtain $\tilde{g}=\tilde{g}(c)$. Following the general approximation scheme presented in section VI we solve instead Eq. (36), which for the exponential distribution case can be written explicitly in the following simpler form

$$
\tilde{g}(2-\tilde{g})\left\{1+\frac{1-\tilde{g}}{(1-\tilde{g}+c \tilde{g})^{2}}+\frac{(1-\tilde{g})^{3 / 2}}{c\left[c+(1-c)(1-\tilde{g})^{3 / 2}\right]}\right\}=1 .
$$

To calculate the parameter $g$, we use Eq. (39). Plugging in the generating function $G_{0}^{1}(x)$ of the exponential degree distribution, given by Eq. (72), we obtain

$$
g=\left[1+\sum_{n=1}^{\infty} \frac{(1-\tilde{g})^{n}}{c-(c-1)(1-\tilde{g})^{n}}\right]^{-1},
$$

where $\tilde{g}$ is given by Eq. (75). In the case of the exponential distribution we have a useful approximation scheme which is similar to the one used in the self-consistent equation for $\tilde{g}$. This amounts to separating the first term from the rest of the sum in Eq. (76), and replacing the sum by an integral. This yields

$$
g=\left[1+\frac{1-\tilde{g}}{c-(c-1)(1-\tilde{g})}+\int_{3 / 2}^{\infty} \frac{(1-\tilde{g})^{n}}{c-(c-1)(1-\tilde{g})^{n}} d n\right]^{-1},
$$

Carrying out the integration, we obtain

$$
g=\left[1+\frac{1-\tilde{g}}{c-(c-1)(1-\tilde{g})}+\frac{\ln \left[1-\left(\frac{c-1}{c}\right)(1-\tilde{g})^{3 / 2}\right]}{(c-1) \ln (1-\tilde{g})}\right]^{-1}
$$

It turns out that this expression is precise within less than one percent compared to the full expression (76), even next to the percolation transition. 


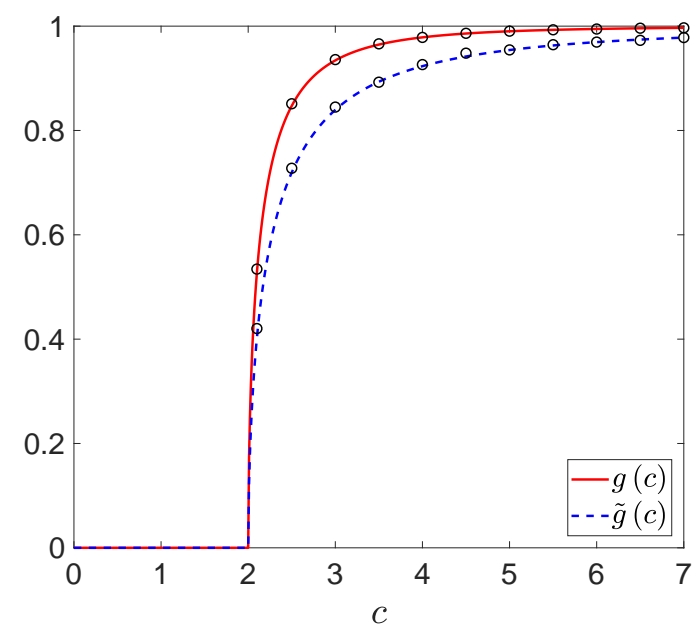

FIG. 2: (Color online) The fraction of nodes, $g$ (solid line), and the fraction of random neighbors of random nodes $\tilde{g}$ (dashed line), that reside on the giant component, in a configuration model network whose giant component exhibits an exponential degree distribution, $P(k \mid 1)$, expressed by Eq. (71), as a function of the mean degree $c=\mathbb{E}[K \mid 1]$ of the giant component. As discussed in the text, the minimal value of the mean degree of the giant component is $c=2$. Thus, for $c<2$ both $g=0$ and $\tilde{g}=0$, while for $c>2$ the parameters $g$ and $\tilde{g}$ quickly increase. The simulation results (circles), obtained for $N=10^{4}$, are in very good agreement with the analytical results.

In order to obtain a single component network of $N_{1}$ nodes with a given exponential degree distribution, $P(k \mid 1)$, one generates a configuration model network with the degree distribution $P(k)$, given by Eq. (21), where $\tilde{g}$ is given by Eq. (175), $g$ is given by Eq. (78) and $P(k \mid 1)$ is given by Eq. (71).

In Fig. 2 we present analytical results for the probability $g$, obtained from Eq. (78), that a randomly selected node resides on the giant component (solid line), in a configuration model network whose giant component exhibits an exponential degree distribution, as a function of the mean degree $c=\mathbb{E}[K \mid 1]$ of the giant component. We also show analytical results for the probability, $\tilde{g}$, obtained from Eq. (75), that a random neighbor of a random node resides on the giant component (dashed line). As in the case of the ternary degree distribution, both $g$ and $\tilde{g}$ vanish for $c<2$, while for $c>2$ they exhibit a steep rise as $c$ is increased. The results of computer simulations (circles) with $N=10^{4}$ are in very good agreement with the analytical results. 
In Fig. 3 we present analytical results (dashed lines) for the degree distributions $P(k)$ and simulation results for the corresponding degree sequences $(\times)$ of the configuration model networks whose giant components exhibit exponential degree distributions with mean degrees $c=\mathbb{E}[K \mid 1]$, where $c=2.1$ (a), $c=2.5$ (b) and $c=3.0$ (c). The degree sequences of the resulting single-component networks (circles) fit perfectly with the desired exponential degree distributions (solid lines), given by Eq. (71). It is found that on the giant component the abundance of nodes of degree $k=1$ is depleted with respect to their abundance in the whole network, while the abundance of nodes of higher degrees is enhanced.

In Fig. 4 we present the mean degree $\langle K\rangle$ (dashed line), obtained from Eq. (40), of a configuration model network whose giant component exhibits an exponential degree distribution with mean degree $c=\mathbb{E}[K \mid 1]$, as a function of $c$. The mean degree, $c$, of the giant component (solid line) is also shown for comparison. It is found that for dilute networks $\langle K\rangle$ is significantly smaller than $c$ and the gap between the two curves shrinks as the network becomes denser. The simulation results (circles), obtained for $N=10^{4}$, are found to be in very good agreement with the analytical results.

\section{Construction of a single component network with a power-law degree distri-} bution

Consider a configuration model network whose giant component exhibits a power-law degree distribution of the form

$$
P(k \mid 1)=\frac{A}{k^{\gamma}},
$$

for $k_{\min } \leq k \leq k_{\max }$. Here we focus on the case of $k_{\min }=1$. In this case, the normalization coefficient is

$$
A=\frac{1}{\zeta(\gamma)-\zeta\left(\gamma, k_{\max }+1\right)}
$$

where $\zeta(s, a)$ is the Hurwitz zeta function and $\zeta(s)=\zeta(s, 1)$ is the Riemann zeta function [30]. In order to avoid correlations, the network size must satisfy the condition $N>\left(k_{\max }\right)^{2} /\langle K\rangle[31$ 33]. The mean degree is given by 

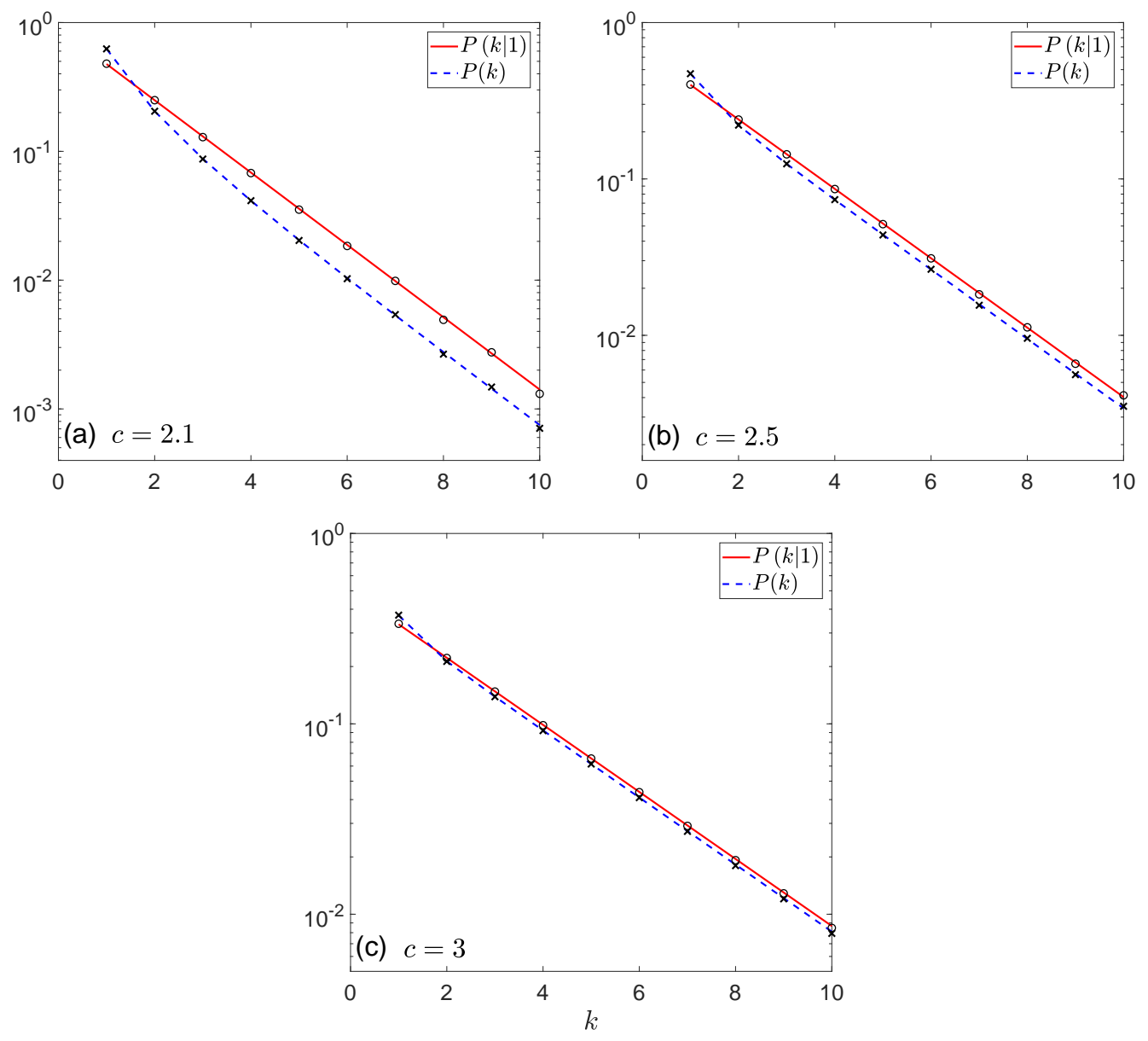

FIG. 3: (Color online) Analytical results (dashed lines) for the degree distributions $P(k)$ and simulation results for the corresponding degree sequences with $N=10^{4}(\times)$, of configuration model networks whose giant components exhibit exponential degree distributions (solid lines) of the form $P(k \mid 1)$, given by Eq. (71), with mean degree $c=\mathbb{E}[K \mid 1]$, where $c=2.1$ (a), $c=2.5$ (b) and $c=3.0(\mathrm{c})$. The degree sequences of the resulting single-component networks (circles) fit perfectly with the desired exponential degree distributions (solid lines). It is found that on the giant component the abundance of nodes of degree $k=1$ is depleted, while the abundance of nodes of higher degrees is slightly enhanced. This feature is most pronounced in the dilute network limit, in which the fraction of nodes that reside on the giant components is small.

$$
c=\mathbb{E}[K \mid 1]=\frac{\zeta(\gamma-1)-\zeta\left(\gamma-1, k_{\max }+1\right)}{\zeta(\gamma)-\zeta\left(\gamma, k_{\max }+1\right)} .
$$

As noted above, a single connected component with a degree distribution $P(k \mid 1)$ exists only if the condition $\mathbb{E}[K \mid 1] \geq 2$ is satisfied. This implies that for a given value of $k_{\max }$ there 


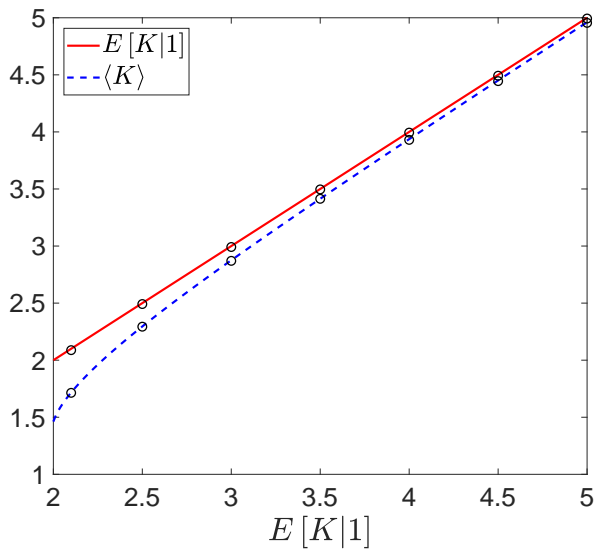

FIG. 4: (Color online) Analytical results (dashed line) and simulation results, obtained for $N=10^{4}$ (circles), for the mean degree $\langle K\rangle$ of a configuration model network whose giant component exhibits an exponential degree distribution with mean degree $c=\mathbb{E}[K \mid 1]$, as a function of $\mathbb{E}[K \mid 1]$. For comparison we also present the analytical results (solid line) and simulation results (circles) for the mean degree $\mathbb{E}[K \mid 1]$ of the giant component. It is found that in the dilute network limit $\langle K\rangle$ is significantly smaller than $c=\mathbb{E}[K \mid 1]$ and the two curves converge as the network becomes denser.

exists a critical value of $\gamma$, denoted by $\gamma_{c}\left(k_{\max }\right)$, such that a giant component exists only for $\gamma<\gamma_{c}\left(k_{\max }\right)$. The value of $\gamma_{c}\left(k_{\max }\right)$ is obtained by solving Eq. (81) for $\gamma$ under the condition that $c=2$. In the special case of $k_{\max } \rightarrow \infty$ one obtains $\gamma_{c}\left(k_{\max }\right) \rightarrow \gamma_{c}(\infty)=3.4787 \ldots$, which is a solution of the equation $\zeta(\gamma-1)=2 \zeta(\gamma)$.

The second moment of the degree distribution is

$$
\mathbb{E}\left[K^{2} \mid 1\right]=\frac{\zeta(\gamma-2)-\zeta\left(\gamma-2, k_{\max }+1\right)}{\zeta(\gamma)-\zeta\left(\gamma, k_{\max }+1\right)} .
$$

For $\gamma \leq 2$, in the asymptotic limit of $N \rightarrow \infty$, the mean degree $\mathbb{E}[K \mid 1]$ diverges in the limit $k_{\max } \rightarrow \infty$. For $2<\gamma \leq 3$, in the asymptotic limit, the mean degree is bounded while the second moment $\mathbb{E}\left[K^{2} \mid 1\right]$ diverges. For $\gamma>3$ both moments are bounded. The generating functions of $P(k \mid 1)$ for a giant component with a power-law degree distribution are

$$
G_{0}^{1}(x)=\frac{\operatorname{Li}_{\gamma}(x)-x^{k_{\max }+1} \Phi\left(x, \gamma, k_{\max }+1\right)}{\zeta(\gamma)-\zeta\left(\gamma, k_{\max }+1\right)}
$$

and 


$$
G_{1}^{1}(x)=\frac{\operatorname{Li}_{\gamma-1}(x)-x^{k_{\max }+1} \Phi\left(x, \gamma-1, k_{\max }+1\right)}{x\left[\zeta(\gamma-1)-\zeta\left(\gamma-1, k_{\max }+1\right)\right]},
$$

where $\operatorname{Li}_{\gamma}(x)$ is the polylogarithmic function. Inserting the expressions for the two generating functions into Eq. (36), we obtain

$$
\begin{aligned}
\tilde{g}(2-\tilde{g}) & {\left[1+(1-\tilde{g}) \frac{\operatorname{Li}_{\gamma-1}(1-\tilde{g})-(1-\tilde{g})^{k_{\max }+1} \Phi\left(1-\tilde{g}, \gamma-1, k_{\max }+1\right)}{(1-\tilde{g})\left[\zeta(\gamma-1)-\zeta\left(\gamma-1, k_{\max }+1\right)\right]}\right.} \\
& \left.-\frac{\operatorname{Li}_{\gamma}\left[(1-\tilde{g})^{3 / 2}-(1-\tilde{g})^{3\left(k_{\max }+1\right) / 2} \Phi\left[(1-\tilde{g})^{3 / 2}, \gamma, k_{\max }+1\right]\right.}{\ln (1-\tilde{g})\left[\zeta(\gamma-1)-\zeta\left(\gamma-1, k_{\max }+1\right)\right]}\right]=1 .
\end{aligned}
$$

This is an implicit equation for $\tilde{g}$ in terms of the exponent $\gamma$ and the upper cutoff $k_{\max }$, that should be solved numerically. The parameter $g$ is then obtained from Eq. (39). Inserting $G_{0}^{1}(x)$ from Eq. (83) into Eq. (39), we obtain

$$
g=\left[1+\sum_{n=1}^{\infty} \frac{\operatorname{Li}_{\gamma}\left[(1-\tilde{g})^{n}\right]-(1-\tilde{g})^{n\left(k_{\max }+1\right)} \Phi\left[(1-\tilde{g})^{n}, \gamma, k_{\max }+1\right]}{\zeta(\gamma)-\zeta\left(\gamma, k_{\max }+1\right)}\right]^{-1}
$$

In order to generate an ensemble of single component networks whose mean size is $\left\langle N_{1}\right\rangle$, which exhibit a given power-law degree distribution $P(k \mid 1)$, one generates configuration model networks of size $N=\left\langle N_{1}\right\rangle / g$ with the degree distribution $P(k)$, given by Eq. (21), where $\tilde{g}$ is given by Eq. (85), $g$ is given by Eq. (86) and $P(k \mid 1)$ is given by Eq. (79). Note that for $\gamma \geq 2$, in the limit of $k_{\max } \rightarrow \infty$ one obtains that $g \rightarrow g_{\infty}<1$. This means that in configuration model networks which exhibit a power-law degree distribution with $\gamma \geq 2$ the giant component does not encompass the whole network regardless of the value of $k_{\max }$. This means that the approach presented here is applicable and useful for the construction of single component random networks with power-law degree distributions for the whole range of $2 \leq \gamma \leq \gamma_{c}(\infty)$.

In Fig. 5 we present analytical results (solid line), obtained from Eq. (81), for the mean degree, $c=\mathbb{E}[K \mid 1]$, of the giant component of a configuration model network, for which the giant component exhibits a power-law degree distribution, $P(k \mid 1)$, given by Eq. (79), as a function of the exponent $\gamma$ for $2<\gamma<2$. . The upper cutoff of the degree distribution is $k_{\max }=100$. The dashed line, presented for $\gamma>2.4$, is still a solution of Eq. (81). However, it does not describe the mean degree of a giant component, because in this regime $c<2$ while the degree distribution of a giant component must satisfy $c>2$. The results for the 


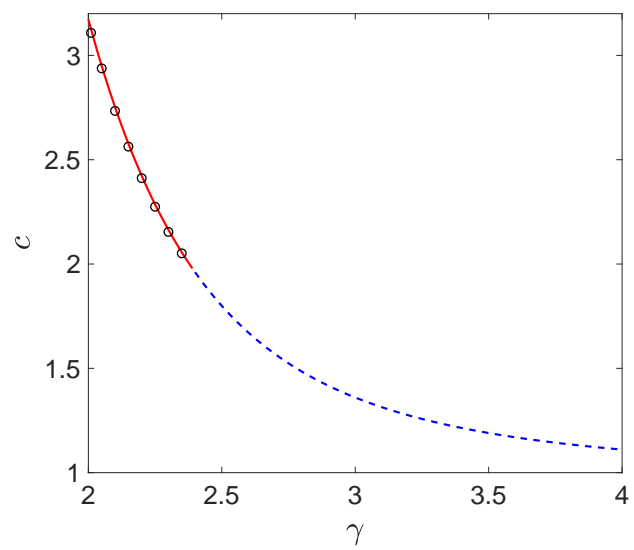

FIG. 5: (Color online) The mean degree, $c=\mathbb{E}[K \mid 1]$, of the giant component of a configuration model network (solid line) with a power-law degree distribution [Eq. (179)], as a function of the exponent $\gamma$, for $\gamma \geq 2$ with $k_{\max }=100$, given by Eq. (81). The mean degree decreases as $\gamma$ is increased. For $\gamma>2.4$ the solid line is replaced by a dashed line, which is still a solution of Eq. (81). However, it does not describe the mean degree of a giant component, because in this regime $c<2$ while the mean degree of a giant component must satisfy $c \geq 2$. The results for the mean degrees of the single component networks constructed using this method (circles) are in perfect agreement with the analytical results.

mean degrees of the network instances constructed using this method (circles) are in perfect agreement with the analytical results. It is found that the mean degree decreases as $\gamma$ is increased.

In Fig. 6 we show analytical results for the values of the parameters $g$ (solid line) and $\tilde{g}$ (dashed line) of a configuration model network whose giant component exhibits a power-law degree distribution, as a function of the mean degree $c=\mathbb{E}[K \mid 1]$ of the giant component. As discussed above, both $g$ and $\tilde{g}$ vanish for $c<2$, since there are no giant components with mean degrees lower than 2. For $c>2$ the parameters $g$ and $\tilde{g}$ gradually increase. This is in contrast to the case of the exponential degree distribution, shown in Fig. 2, in which $g$ and $\tilde{g}$ increase more steeply. The simulation results (circles) for $g$, obtained from network instances constructed using this method with $k_{\max }=100$ and $N=4 \times 10^{4}$ are found to be in good agreement with the analytical results, while the results for $\tilde{g}$ are a bit noisy.

In Fig. 7 we present analytical results (dashed lines) for the degree distributions $P(k)$ 


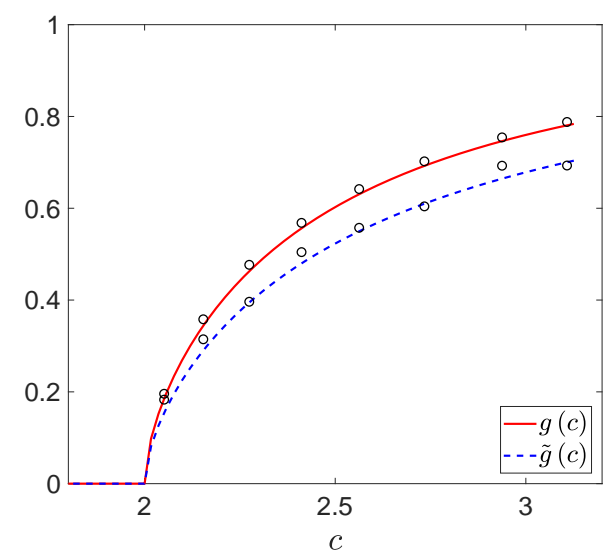

FIG. 6: (Color online) The parameters $g$ (solid line) and $\tilde{g}$ (dashed line) of a configuration model network whose giant component exhibits a power-law degree distribution of the form $P(k \mid 1)$, given by Eq. (179), as a function of the mean degree $c=\mathbb{E}[K \mid 1]$ of the giant component. As discussed in the text the minimal value of the mean degree of a giant component with a power-law degree distribution is $c=2$. Thus, for $c<2$ both $g=0$ and $\tilde{g}=0$. For $c>2$ the parameters $g$ and $\tilde{g}$ gradually increase. This is in contrast to the case of the exponential degree distribution, shown in Fig. 2, in which $g$ and $\tilde{g}$ increase more steeply.

[given by Eq. (21), where $\tilde{g}$ is the solution of Eq. (85) and $g$ is given by Eq. (86)] and simulation results for the corresponding degree sequences $(x)$ of the configuration model networks whose giant components exhibit power-law degree distributions, with $\gamma=2.01$ (a), $\gamma=2.2$ (b) and $\gamma=2.35$ (c). The degree sequences of the resulting single-component networks (circles) fit perfectly with the desired power-law distributions (solid lines), given by Eq. (79).

In Fig. 8 we present analytical results (dashed line) for the mean degree $\langle K\rangle$ of a configuration model network whose giant component exhibits a power-law degree distribution, given by Eq. (79) with $k_{\max }=100$, as a function of the mean degree $c=\mathbb{E}[K \mid 1]$ of the giant component. The mean degree $c$ of the giant component (solid line), is also shown for comparison. It is found that in the dilute network limit $\langle K\rangle$ is much smaller than $c=\mathbb{E}[K \mid 1]$. The gap between the two curves slightly decreases as the network becomes more dense, but the two curves do not converge. This is due to the fact that even for the largest value of $\mathbb{E}[K \mid 1]$ that can be obtained with $k_{\max }=100$ the giant component does not encompass the 

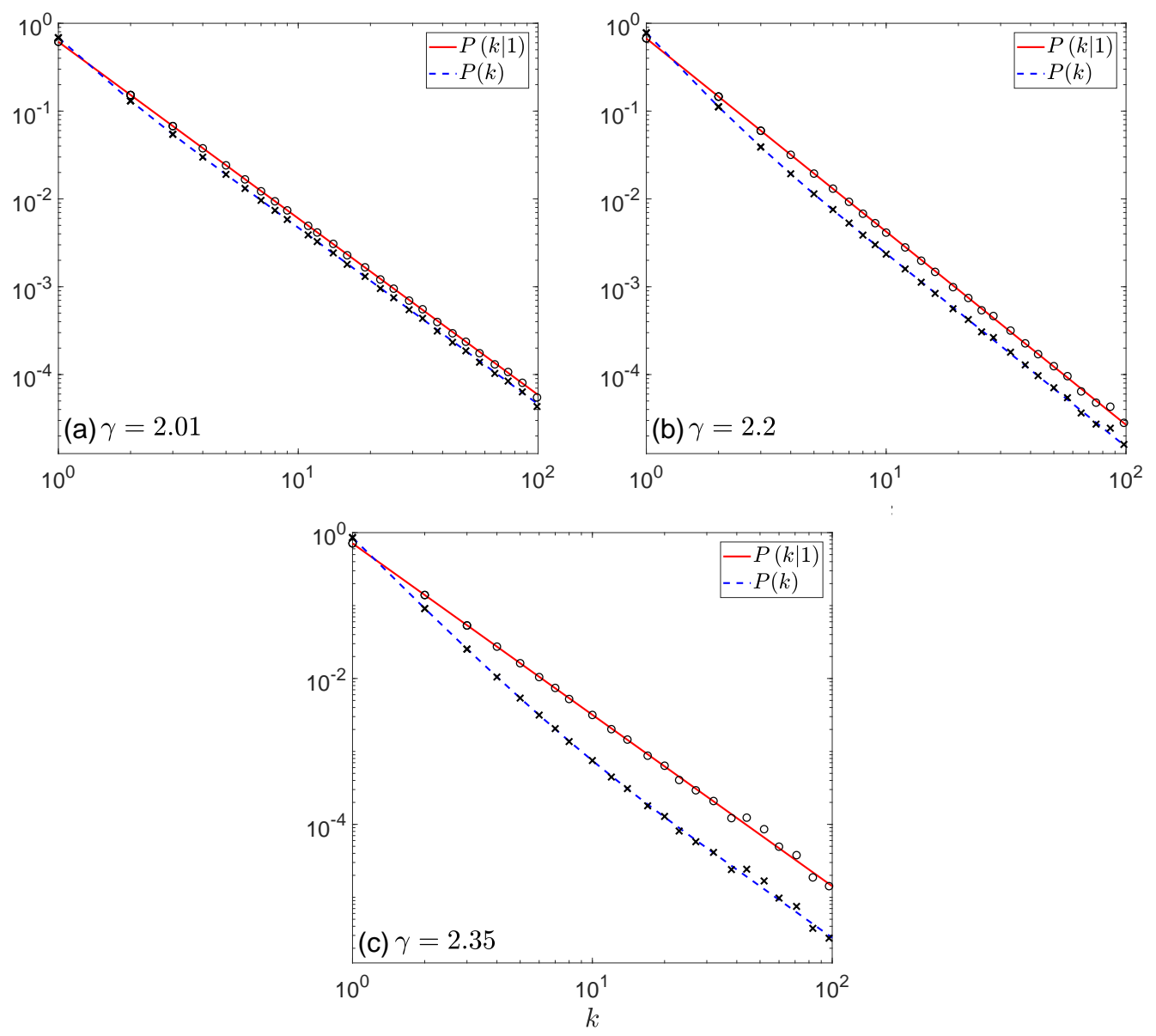

FIG. 7: (Color online) Analytical results (dashed lines) for the degree distributions $P(k)$ and simulation results with $N=4 \times 10^{4}$ for the corresponding degree sequences $(\times)$ of configuration model networks whose giant components exhibit power-law degree distributions (solid lines), of the form $P(k \mid 1)$, given by Eq. (79), with $\gamma=2.01$ (a), $\gamma=2.2$ (b) and $\gamma=2.35$ (c), and with $k_{\max }=100$. The degree sequences of the resulting single-component networks (circles), fit perfectly with the desired power-law degree distributions (solid lines). It is found that on the giant component the abundance of nodes of degree $k=1$ is depleted, while the abundance of nodes of higher degrees is enhanced. This feature is most pronounced in the dilute network limit, in which the fraction of nodes that reside on the giant components is small.

whole network. The gap between $\langle K\rangle$ can be decreased further by increasing the value of $k_{\max }$. However, in order to maintain the whole network uncorrelated its size $N$ should satisfy $N>\left(k_{\max }\right)^{2} /\langle K\rangle[31-33]$. The results obtained from computer simulations (circles) with $N=4 \times 10^{4}$ are found to be in very good agreement with the analytical results. 


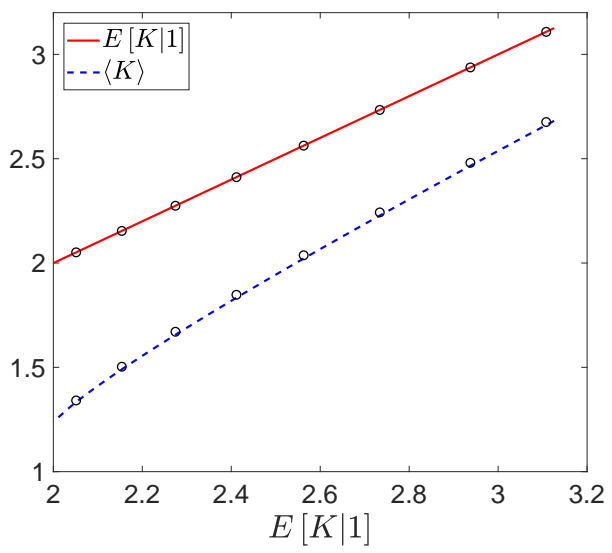

FIG. 8: (Color online) The mean degree $\langle K\rangle$ of a configuration model network whose giant component exhibits a power-law degree distribution with mean degree $c=\mathbb{E}[K \mid 1]$, as a function of $\mathbb{E}[K \mid 1]$ (dashed line). The mean degree $\mathbb{E}[K \mid 1]$ of the giant component (solid line), is also shown for comparison. It is found that in the dilute network limit $\langle K\rangle$ is much smaller than $\mathbb{E}[K \mid 1]$. The gap between the two curves slightly decreases as the network becomes more dense, but the two curves do not converge. The simulation results (circles), obtained for $N=4 \times 10^{4}$, are in very good agreement with the analytical results.

\section{DISCUSSION}

While configuration model networks are random and uncorrelated, their giant components exhibit correlations between the degrees of adjacent nodes. These degree-degree correlations and the assortativity coefficients of the giant components were studied in Ref. [17]. The giant components were found to be disassortative, namely high-degree nodes tend to connect preferentially to low-degree nodes and vice versa. Moreover, it was found that as the network approaches the percolation transition from above and the giant component decreases in size, its structure becomes more distinct from the structure of the overall network. In particular, the degree distribution of the giant component deviates more strongly from the degree distribution of the whole network, the degree-degree correlations become stronger and the assortativity coefficient becomes more negative.

The disassortativity of the giant component helps to maintain its integrity. For example, the probability of a pair of nodes of degrees $k, k^{\prime}=1$, which reside on the giant component, to connect to each other must vanish, otherwise they will form an isolated dimer. This 
means that nodes of degree $k=1$ preferentially connect to nodes of higher degrees. As a result, high-degree nodes preferentially connect to nodes of degree $k=1$. In fact, the giant component exhibits degree-degree correlations of all orders. These correlations are required in order to exclude the possibility that a randomly selected node belongs to an isolated component of any finite size [17]. Interestingly, disassortativity was found to be prevalent in a broader class of scale-free networks which exhibit correlations and can be explained by entropic considerations [34, 35].

The methodology introduced in this paper enables the construction of random networks that consist of a single connected component of $N_{1}$ nodes with a given degree distribution $P(k \mid 1)$. The desired network consists of the giant component of a suitable configuration model network of $N$ nodes and degree distribution $P(k)$. For a given value of $N$ the size $N_{1}$ of the giant component exhibits fluctuations which satisfy $\operatorname{Var}\left(N_{1}\right) \propto N$, which are thus under control in the asymptotic limit. We also present an adjustment procedure for the case in which a specific value of $N_{1}$ is required.

The construction of random networks that consist of a single connected component with a given degree distribution is expected to be useful for the analysis of empirical networks. A common practice in the study of empirical networks is to generate an ensemble of randomized networks with the same degree sequence as the empirical network. One then compares structural and statistical properties of the empirical network to the corresponding properties of the randomized networks. The differences between the empirical network and its randomized counterparts may imply some significant functional or evolutionary properties of the empirical network. Stated more technically, randomized networks serve as null models for empirical networks [31, 32, 36-40]. This approach was utilized in the study of network motifs, which are over-represented in empirical networks compared to the corresponding randomized networks [41, 42]. It was also used in the analysis of degree-degree correlations, the assortativity coefficient and the clustering coefficient [43-45], and in the study of the distribution of shortest path lengths [46].

A randomized network with the same degree sequence as a given empirical network can be constructed in two different ways. One way is to generate a configuration model network with the given degree sequence obtained from the empirical network. Another way is to start from the empirical network and apply a series of rewiring steps. In each rewiring step one picks two random edges, $i-j$ and $i^{\prime}-j^{\prime}$ and then exchanges them such that $i$ becomes 
connected to $j^{\prime}$ and $i^{\prime}$ becomes connected to $j$. In a case in which either the $i-j^{\prime}$ edge or the $i^{\prime}-j$ edge already exists the step is rejected. After a large number of such rewiring steps one obtains a randomized network which maintains the degree sequence of the empirical network.

In some cases one may be interested in finding the degree distribution from which the given degree sequence of the empirical network is most likely to arise. Consider an empirical network of $N$ nodes, whose degree sequence is given by $\left\{n_{k}^{\mathrm{E}}\right\}, k=1,2, \ldots, k_{\max }$, where $n_{k}^{\mathrm{E}}$ is the number of nodes of degree $k$ and $\sum_{k} n_{k}^{\mathrm{E}}=N$. The degree distribution from which this degree sequence is most likely to emerge is given by

$$
P(k)=\frac{n_{k}^{\mathrm{E}}}{N},
$$

where $k=1,2, \ldots, k_{\max }$. Sampling the degrees of $N$ nodes from this distribution, the probability to obtain a degree sequence of the form $\left\{n_{k}\right\}, k=1,2, \ldots, k_{\max }$ is

$$
P\left(\left\{n_{k}\right\}\right)=\frac{N !}{\prod_{k=1}^{k_{\max }} n_{k} !} \prod_{k=1}^{k_{\max }} P(k)^{n_{k}} .
$$

Configuration model networks with degree sequences that are drawn from the degree distribution $P(k)$, given by Eq. (87), provide a broader class of randomized networks for the given empirical networks. While their degree sequences are not identical to the degree sequence of the empirical network their statistical properties are closely related. This is a grand-canonical approach to the sampling problem.

While some empirical networks consist of a single connected component such as transportation networks and brain networks [47], other networks consist of many isolated components of various sizes such as adoption of innovations or products networks [48] and mobile phone calling networks [49]. The distribution of sizes of these components has been studied in the context of subcritical networks and provides a useful characterization of the network structure [24]. In a case in which one of the isolated components is particularly large (and asymptotically encompasses a macroscopic fraction of the network size), it is referred to as the giant component. In such case the network exhibits a coexistence between the giant component and many finite components. Here we focus on the properties of the giant component, namely the degree distribution, degree-degree correlations, clustering coefficient and size. The size of the giant component, $N_{1}$, depends on the size of the whole network, $N$, 
and on the fraction of nodes, $0<g<1$, that reside on the giant component. In computer simulations the value of $g$ varies between different network instances in a given network ensemble, following a distribution $P(g)$ that is characteristic of the given ensemble. In empirical networks it is difficult to find many network instances that are drawn from the same statistical ensemble. Therefore, it is difficult to find a direct analog of $P(g)$ in empirical networks.

In a case in which the empirical network under study consists of a single connected component, it is desirable that the corresponding randomized networks will also consist of a single connected component. The procedures described above may produce randomized networks that consist of multiple components (such as a giant component and many finite components), even in a case in which the empirical network consists of a single connected component. The size of the giant component of the randomized network depends on its degree sequence and can be determined using methods of percolation theory.

The methodology presented in this paper provides a way to obtain a randomized network that consists of a single connected component. Consider an empirical network of $N_{1}$ nodes that consists of a single connected component with degree sequence $\left\{n_{k}\right\}$. Using Eq. (87) one obtains the most probable degree distribution $P(k \mid 1)$ for the given degree sequence. Using the procedure presented in this paper, one obtains the size $N$ and the degree distribution $P(k)$ of a configuration model network whose giant component is the desired randomized network.

\section{SUMMARY}

We presented a method for the construction of ensembles of random networks that consist of a single connected component of any desired size $N_{1}$ with a pre-defined degree distribution $P(k \mid 1)$. The construction is done by generating a configuration model network with a suitable degree distribution $P(k)$ and size $N$, whose giant component is of size $N_{1}$ and its degree distribution is $P(k \mid 1)$. This approach is based on the inversion of the relation between $P(k)$ and $P(k \mid 1)$, which was presented in Ref. [17]. It extends the construction toolbox of random networks beyond the configuration model framework, in which one controls the network size and the degree distribution but has no control over the number of network components and their sizes. The capability of generating single component random networks 
with a desired degree distribution is expected to be instrumental in the effort to elucidate the statistical properties of such networks at the local and global scales.

[1] R. Albert and A.-L. Barabási, Statistical mechanics of complex networks, Rev. Mod. Phys. 74, 47 (2002).

[2] S.N. Dorogovtsev and J.F.F. Mendes, Evolution of networks: From biological networks to the Internet and $W W W$, (Oxford University Press, Oxford, 2003).

[3] S.N. Dorogovtsev, A.V. Goltsev and J.F.F. Mendes, Critical phenomena in complex networks, Rev. Mod. Phys. 80, 1275 (2008).

[4] R. van der Hofstad, Random graphs and complex networks (Eindhoven, 2013); Available at https://www.win.tue.nl/ rhofstad/NotesRGCN2013.pdf

[5] M.E.J. Newman, Networks: An introduction, (Oxford University Press, Oxford, 2010).

[6] S. Havlin and R. Cohen, Complex networks: Structure, robustness and function, (Cambridge University Press, New York, 2010).

[7] E. Estrada, The structure of complex networks: theory and applications, (Oxford University Press, Oxford, 2011).

[8] A. Barrat, M. Barthélemy and A. Vespignani, Dynamical processes on complex networks, (Cambridge University Press, Cambridge, 2012).

[9] V. Latora, V. Nicosia and G. Russo, Complex Networks: Principles, Methods and Applications, (Cambridge University Press, Cambridge, 2012).

[10] P. Erdős and A. Rényi, On random graphs I, Publicationes Mathematicae 6, 290 (1959).

[11] P. Erdős and A. Rényi, On the evolution of random graphs, Publ. Math. Inst. Hung. Acad. Sci. 5, 17 (1960).

[12] P. Erdős and A. Rényi, On the evolution of random graphs II, Bull. Inst. Int. Stat. 38, 343 (1961).

[13] B. Bollobás, The evolution of random graphs, Trans. Amer. Math. Soc. 286, 257 (1984).

[14] M. Molloy and A. Reed, A critical point for random graphs with a given degree sequence, Random Structures and Algorithms 6, 161 (1995).

[15] M. Molloy and A. Reed, The size of the giant component of a random graph with a given degree sequence, Combin., Prob. and Comp. 7, 295 (1998). 
[16] B. Bollobás, Random graphs (Cambridge University Press, Cambridge, 2001).

[17] I. Tishby, O. Biham, E. Katzav and R. Kühn, Revealing the micro-structure of the giant component in random graph ensembles, Phys. Rev. E 97, 042318 (2018).

[18] M.E.J. Newman, S.H. Strogatz and D.J. Watts, Random graphs with arbitrary degree distributions and their applications, Phys. Rev. E 64, 026118 (2001).

[19] R. Cohen and S. Havlin, Scale-free networks are ultrasmall, Phys. Rev. Lett. 90, 058701 (2003).

[20] P. Erdős and T. Gallai, Gráfok előírt fokszámú pontokkal, Matematikai Lapok 11, 264 (1960).

[21] S.A. Choudum, A simple proof of the Erdős-Gallai theorem on graph sequences, Bulletin of the Australian Mathematical Society 33, 67 (1986).

[22] H. Bonneau, A. Hassid, O. Biham, R. Kühn and E. Katzav, Distribution of shortest cycle lengths in random networks, Phys. Rev. E 96, 062307 (2017).

[23] I. Tishby, O. Biham, R. Kühn and E. Katzav, Statistical analysis of articulation points in configuration model networks, Phys. Rev. E 98, 062301 (2018).

[24] E. Katzav, O. Biham, and A.K. Hartmann, Distribution of shortest path lengths in subcritical Erdős-Rényi networks, Phys. Rev. E. 98, 012301 (2018).

[25] M. Kang, Giant components in random graphs, The IMA Volumes in Mathematics and its Applications 159, page 235, edited by A. Beveridge et al. (Springer International Publishing Switzerland, 2016).

[26] B. Bollobas and O. Riordan, The phase transition in the Erdős-Rényi random graph process, Bolyai Society Mathematical Studies 25, 59 (2013).

[27] O. Riordan, The phase transition in the configuration model, Combinatorics, Probability and Computing 21, 265 (2012).

[28] S. Mizutaka and T. Hasegawa, Disassortativity of percolating clusters in random networks, Phys. Rev. E 98, 062314 (2018).

[29] M.E.J. Newman, Assortative mixing in networks, Phys. Rev. Lett. 89, 208701 (2002).

[30] F.W.J. Olver, D.M. Lozier, R.F. Boisvert and C.W. Clark, NIST handbook of mathematical functions (Cambridge University Press, Cambridge, 2010).

[31] G. Bianconi, The entropy of randomized network ensembles, Europhys. Lett. 81, 28005 (2008).

[32] G. Bianconi, Entropy of network ensembles, Phys. Rev. E 79, 036114 (2009).

[33] A.J.E.M. Janssen and J.S.H. van Leeuwaarden, Giant component sizes in scale-free networks with power-law degrees and cutoffs, EPL 112, 68001 (2015). 
[34] S. Johnson, J.J. Torres, J. Marro and M.A. Munoz, Entropic origin of disassortativity in complex networks, Phys. Rev. Lett. 104, 108702 (2010).

[35] O. Williams and C.I. Del Genio, Degree Correlations in Directed Scale-Free Networks, Plos One 9, e110121 (2014).

[36] T. Coolen, A. Annibale and E. Roberts, Generating Random Networks and Graphs, (Oxford University Press, Oxford, 2017).

[37] A.C.C. Coolen, A. De Martino and A. Annibale, Constrained Markovian dynamics of random graphs, J. Stat. Phys. 136, 1035 (2009).

[38] A. Annibale, A.C.C. Coolen, L.P. Fernandes, F. Fraternali and J. Kleinjung, Tailored graph ensembles as proxies or null models for real networks I: tools for quantifying structure, $J$. Phys. A 42, 485001 (2009).

[39] E.S. Roberts, T. Schlitt and A.C.C. Coolen, Tailored graph ensembles as proxies or null models for real networks II: results on directed graphs, J. Phys. A 44, 275002 (2011).

[40] E.S. Roberts, A. Annibale and A.C.C. Coolen, Tailored Random Graph Ensembles, J. Phys.: Conf. Ser. 410, 012097 (2013).

[41] S.S. Shen-Orr, R. Milo, S. Mangan and U. Alon, Network motifs in the transcriptional regulation network of Escherichia coli, Nature Genetics 31, 64 (2002)

[42] N. Kashtan, S. Itzkovitz, R. Milo and U. Alon, Topological generalizations of network motifs, Phys. Rev. E 70, 031909 (2004).

[43] S. Maslov, K. Sneppen and A. Zaliznyak, Detection of topological patterns in complex networks: correlation profile of the internet, Physica A 333, 529 (2004).

[44] J. Park and M.E.J. Newman, Origin of degree correlations in the Internet and other networks, Phys. Rev. E 68, 026112 (2003).

[45] P. Holme and J. Zhao, Exploring the assortativity-clustering space of a networks degree sequence, Phys. Rev. E 75, 046111 (2007).

[46] L. Giot et al., A Protein Interaction Map of Drosophila melanogaster, Science 302, 1727 (2003).

[47] S. Wandelt, X. Sun, E. Menasalvas, A. Rodriguez-González and M. Zanin, On the use of random graphs as null model of large connected networks, Chaos, Solitons and Fractals 119, 318 (2019).

[48] M. Karsai, G. Iniguez, R. Kikas, K. Kaski and J. Kertész, Local cascades induced global con- 
tagion: how heterogeneous thresholds, exogenous effects, and unconcerned behaviour govern online adoption spreading, Scientific Reports 6, 27178 (2016).

[49] M.-X. Li, Z.-Q. Jiang, W.-J. Xie, S. Micciche, M. Tumminello, W.-X. Zhou and R.N. Mantegna, A comparative analysis of the statistical properties of large mobile phone calling networks, Scientific Reports 4, 5132 (2014). 